\title{
Survivin Antigen Vaccine DPX-Survivac
}

National Cancer Institute

\section{Source}

National Cancer Institute. Survivin Antigen Vaccine DPX-Survivac. NCI Thesaurus. Code C97951.

A lipid depot-based therapeutic cancer vaccine composed of survivin epitopes, a universal T Helper peptide and a polynucleotide adjuvant encapsulated in liposomes and then formulated in the hydrophobic carrier Montanide ISA51 VG, with potential immunopotentiating and antineoplastic activities. Upon injection of the survivin antigen vaccine DPX-Survivac, a depot is created at the injection site from which the antigens and adjuvant are released. This vaccine may elicit a long lasting cellular response ag ainst survivin-expressing cancers, resulting in a decrease in tumor cell proliferation and an induction of tumor cell death. Survivin, a member of the inhibitor of apoptosis (IAP) family expressed during embryonic development, is upregulated in a variety of human cancers while absent in most normal adult cells; its expression in tumors is associated with a more aggressive phenotype, decreased survival, and increased resistance to chemotherapy. 